\title{
Schistosome vaccine testing: lessons from the baboon model
}

\author{
Patricia S Coulson/ ${ }^{+}$, Thomas M Kariuki* \\ Department of Biology, University of York, York YO10 5YW, United Kingdom *Institute of Primate Research, \\ Karen, Nairobi, Kenya
}

The high level of protection elicited in rodents and primates by the radiation-attenuated schistosome vaccine gives hope that a human vaccine relying on equivalent mechanisms is feasible. In humans, a vaccine would be undoubtedly administered to previously or currently infected individuals. We have therefore used the olive baboon to investigate whether vaccine-induced immunity is compromised by a schistosome infection. We showed that neither a preceding infection, terminated by chemotherapy, nor an ongoing chronic infection affected the level of protection. Whilst IgM responses to vaccination or infection were short-lived, IgG responses rose with each successive exposure to the vaccine. Such a rise was obscured by responses to egg deposition in already-infected animals. In human trials it would be necessary to use indirect estimates of infection intensity to determine vaccine efficacy. Using worm burden as the definitive criterion, we demonstrated that the surrogate measures, fecal eggs, and circulating antigens, consistently overestimated protection. Regression analysis of the surrogate parameters on worm burden revealed that the principal reason for overestimation was the threshold sensitivity of the assays. If we extrapolate our findings to human schistosomiasis mansoni, it is clear that more sensitive indirect measures of infection intensity are required for future vaccine trials.

Key words: Schistosoma mansoni - radiation-attenuated vaccine - infection - circulating antigens

A schistosome vaccine would provide a useful tool for the control and eradication of Schistosoma mansoni. In spite of several decades of research an effective vaccine remains elusive but the successful induction of high levels of protective immunity in laboratory hosts, by radiation-attenuated (RA) cercariae, nevertheless gives hope that it is feasible (Coulson 1997). Current advances in post-genomic techniques are providing new avenues to identify the secreted and surface-exposed antigens that mediate protection (Curwen et al. 2004, Dillon et al. 2006) and should eventually lead to replacement of the RA vaccine with recombinant protein formulations. Once their protective potential has been established in laboratory models, human vaccine trials will be required. Such trials will inevitably be undertaken in endemic areas where many people receiving the vaccine will either harbor a schistosome infection or have previously been infected and had curative chemotherapy. Given the fact that schistosome infections rapidly downmodulate from the acute to the chronic stage (King 2001), is it likely that vaccine-induced immunity would be severely compromised in human hosts?

There is a second question mark over human vaccine trials. How effectively can infection intensity be assessed using currently available surrogate measures of worm burden, namely fecal egg output and circulating antigen

Financial support: INCO-DEV Program, Research for Development EC contract ICA4-CT99-10006. TMK received financial assistance from UNICEF/UNDP/World Bank/WHO Special Programme for Research and Training in Tropical Diseases (TDR)

${ }^{+}$Corresponding author: psc3@york.ac.uk

Received 25 May 2006

Accepted 26 June 2006 levels? To date, there have been no convincing studies that accurately describe the relationship between these parameters and worm burden in humans or animal models.

\section{The baboon as a model host for schistosomes}

The olive baboon (Papio anubis) is a highly permissive host for $S$. mansoni, with up to $80 \%$ of penetrant cercariae maturing into adult worms (Yole et al. 1996, Farah et al. 1997) compared with a maximum of $30-40 \%$ in mice. The higher proportion of parasites maturing, the greater the confidence a researcher can have that any reduction associated with vaccination is the result of immune-mediated elimination and not simply some physiological constraint. Another disadvantage of the mouse model is that the egg-induced pathology, resulting from a burden of only one to three worm pairs, leads to significant morbidity and mortality from 12 weeks after infection (McHugh et al. 1987), precluding long-term experiments. In contrast, an adolescent baboon is equivalent in size to a human child and can harbor a substantial burden of several hundred worms indefinitely. The pathology is less intense than in humans but significant periportal fibrosis has been reported in longer-term infections (Farah et al. 2000). The baboon is also phylogenetically closer to humans that the laboratory mouse so that findings are likely to have more relevance to the human condition.

The RA schistosome vaccine is effective in baboons but multiple exposures are needed to elicit a high level of protection (Yole et al. 1996, Kariuki et al. 2004). Indeed, it appears that successive exposures progressively boost schistosome-specific IgG levels but this response is not sustained without continued immunostimulation. Thus, when challenge was given 12 weeks after the last vaccination instead of after the usual three-week interval, a substantial reduction in the level of protection was observed (54 and 86\%, respectively; Kariuki et al. 2004). It is noteworthy that in these experiments the immune priming 
that follows egg deposition greatly exceeds that elicited by attenuated cercariae, in terms of the magnitude of proliferation, cytokine production and ensuing antibody levels. Although the level of anti-larval IgG appears crucial to the protective response, we know little about the immune mechanism(s) involved in parasite elimination in the baboon, or the relevant antigens. While the RA vaccine is unsuitable for use in humans, the fact that it can reproducibly elicit high levels of protection makes it an ideal system for investigating the interaction of vaccination with infection. In this respect, the capacity of the baboon to tolerate long-term infections allows experiments that would never be feasible in the mouse to be undertaken.

\section{Does a schistosome infection compromise vaccine efficacy?}

We have investigated the impact of previous and current schistosome infections on the outcome of exposure to the RA vaccine (Kariuki et al. 2006). In the first experiment we tested whether vaccine efficacy was compromised when the vaccination regime followed closely upon a primary infection that had just reached the chronic downmodulatory stage before being terminated by praziquantel treatment (at 12 and 13 weeks). This required four groups of baboons, two serving as vaccination control and challenge control, respectively. The remaining two comprised infected, treated and vaccinated animals and infected, treated only animals. The vaccination regime comprised five exposures to 9000 attenuated cercariae at four-week intervals. All four groups received a challenge with 800 normal cercariae at 37 weeks (three weeks after the last vaccination), and worms were recovered by perfusion of the hepatic portal system ten weeks after challenge. There was no difference in the level of protection induced in the infected, treated, vaccinated group relative to the vaccinated only control (75 and 77\%, respectively). In parenthesis, infection and treatment alone elicited a modest level of protection, similar to that observed in another recent baboon study (Nyindo et al. 1999).

In the second experiment, we investigated whether vaccine efficacy was compromised when the five vaccinations were superimposed on a chronic schistosome infection over the period from 14 to 30 weeks. This involved three groups, the vaccine-only controls, challenge controls and the infected, vaccinated and treated animals. Chemotherapy was required to eliminate the primary worms before challenge, to permit accurate determination of challenge worm burdens; this was carried out at 32 and 33 weeks prior to challenge at 37 weeks. Once again, the level of protection in the infected, vaccinated group was virtually identical to that of the vaccine-only controls (66 versus $62 \%$ ). The slightly lower levels compared to the first experiment are most likely the result of the longer interval (seven weeks) between the last vaccination and challenge.

It should be noted that in both experiments estimation of fecal egg output and circulating antigens in infected animals confirmed the presence of substantial primary worm burdens prior to curative chemotherapy.

\section{Immune reactivity to infection and vaccination}

We used antibody responses to various crude parasite preparations as indicators of immune reactivity. In groups receiving an initial infection, after an early peak IgM went into a rapid decline that was not boosted by vaccination. By the time of challenge, levels were low and unlikely to contribute to the protective mechanism. This low level of specific IgM persisted after challenge in previously infected groups in spite of significant egg deposition between five and ten weeks. In contrast, both vaccine and challenge controls mounted a strong but not sustained IgM response to eggs. Consecutive vaccinations boosted the IgG response in the vaccine-only group which was also perceptible in the infected, treated and vaccinated group. However, the sustained IgG levels in the group with an ongoing infection obscured any responses to the superimposed vaccination regime. It was notable that, as in previous studies (Yole et al. 1996, Kariuki et al. 2004) the challenge infection failed to boost IgG responses, which continued to decline until the onset of egg deposition. We conclude that the antibody response to schistosome eggs is so dominant that it is difficult to discern any parallel response to other life-cycle stages. The problem is compounded by the degree of antigenic cross-reactivity between larval and egg secretions, largely due to shared glycan epitopes (Eberl et al. 2001). It highlights the need to identify stage-specific indicator antigens that provoke a measurable response after infection or vaccination, before we are able to dissect immune responses to cercariae, schistosomula, adults and eggs.

\section{Use of surrogate measures to determine vaccine efficacy}

The most desirable attribute of a schistosome vaccine would be its ability to reduce worm burdens. In experimental animal models this is reliably determined by comparing values in test and control groups after worm recovery by portal perfusion. Obviously, it is impossible to obtain this direct measure from human patients so that estimates of worm burden must rely on surrogates. Conventionally, these are fecal egg output (Sturrock et al. 1978) and the level of schistosome antigens in the circulation (Deelder et al. 1989, Polman et al. 2000), parameters that would have to be used in any future trial of a vaccine against schistosomiasis mansoni. In our recent baboon studies (Kariuki et al. 2004, 2006), we were able to compare the estimates of protection based on worm burden with those derived from fecal eggs and circulating antigens. We found that in almost all instances the surrogates overestimated the actual level of protection achieved. Figure depicts original data to illustrate the point.

\section{Implications for human schistosomiasis mansoni}

It is important to ask why the surrogates should consistently overestimate protection, a question we shall address in a future publication. However, a major contributory factor is undoubtedly the limited sensitivity of the surrogate assays. A regression of fecal egg output and circulating antigen levels, at steady state prior to per- 

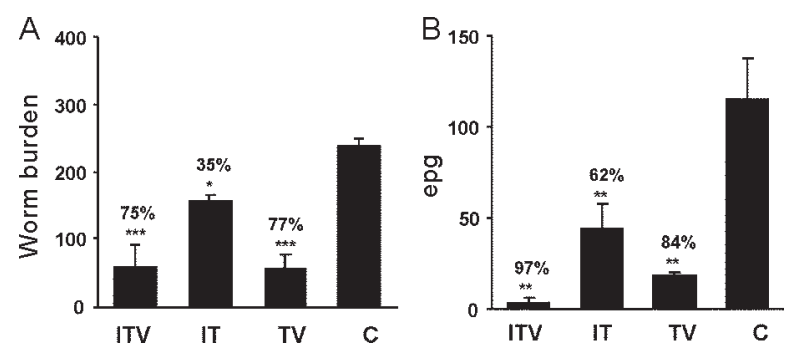

Comparison of the levels of protection calculated from A: worm burdens and B: fecal egg output. Worms were recovered by perfusion of the portal vasculature 10 weeks post-challenge. Values for fecal egg output are the means of eggs per gram (epg) at weeks 8, 9, 10 post-challenge ( 9 replicates). Percent protection was determined using the formula (control - test)/control x 100, and is indicated above each bar; $* * * P<0.001, * * P<0.01, * P<0.05$. Values are mean + SEM $(\mathrm{n}=5-7)$. Experimental groups were ITV (infected, treated, and vaccinated), IT (infected and treated), TV (treated and vaccinated), and $\mathrm{C}$ (challenge control).

fusion, against worm burden revealed thresholds for detection lying between 24 and 47 worms. In the case of fecal eggs, the estimates were based on nine replicate samples per animal, far more than would be made in comparable human studies; for circulating antigens, the values were derived from two or three samples per animal. If we extrapolate from our findings to the human situation, the impact of assay insensitivity will be greatest where people have low worm burdens, leading to a larger proportion of individuals being considered negative than is warranted by their actual infection status. Thus, chemotherapy of only those people with a positive fecal smear or circulating antigen assay will miss a proportion of the population who are contributing to transmission. Furthermore, reliance on surrogates for evaluation of treatment will result in overconfidence about its efficacy. A method for accurately determining infection intensity is crucial, in view of the recent demonstration by King et al. that the performance-related symptoms and disability linked morbidities due to schistosomiasis are greater than originally estimated (King et al. 2005). This study concluded that even light infections should not be overlooked as a cause of disability.

These observations also have implications for vaccine testing. Human vaccine trials are likely to be undertaken in individuals given chemotherapy to "clear" their worms, and ultimately in previously unexposed children. After administration of the vaccine or placebo, the worm burdens that will gradually accrue in these individuals will only become reliably detectable as they rise above the thresholds. Although the threshold distortion diminishes with higher worm burden, protection will always be overestimated. The consequence would be a misleading impression that the vaccine was more efficacious than in reality, especially if the evaluation was carried out soon after egg excretion began in the placebo group.

\section{Conclusions}

Given both the ethical concerns and the expense of using primates for research, it is pertinent to ask what we have been able to achieve in the baboon that would not have been possible in the murine host. Our most important finding was that the protection elicited by the RA vaccine is not diminished by either a previous or an ongoing schistosome infection. From this we infer that the vaccine-induced immunological mechanisms are not compromised by the infection in spite of the fact that antibody responses to eggs, measured using crude antigen preparations, totally obscure those to larvae. The dissociation of vaccine- from egg-induced responses augers well for the success of a recombinant vaccine based on the mechanisms of the RA vaccine. We have also established, for the first time, the quantitative relationship between worm burden and the surrogates used to estimate infection intensity, so revealing the limitations of the latter. This emphasizes the need for more sensitive determinants of infection intensity before human trials of schistosomiasis mansoni vaccines take place. The requirement for better assays is not a novel sentiment, having been expressed by de Vlas and Gryseels in 1992, but there have been no dramatic improvements in the intervening years.

\section{REFERENCES}

Coulson PS 1997. The radiation-attenuated vaccine against schistosomes in animal models: paradigm for a human vaccine? Adv Parasitol 39: 271-336.

Curwen RS, Ashton PD, Johnston DA, Wilson RA 2004. The Schistosoma mansoni soluble proteome: a comparison across four life-cycle stages. Mol Biochem Parasitol 138: 57-66.

de Vlas SJ, Gryseels B 1992. Underestimation of Schistosoma mansoni prevalences. Parasitol Today 8: 274-277.

Deelder AM, De Jonge N, Boerman OC, Fillie YE, Hilberath GW, Rotmans JP, Gerritse MJ, Schut DW 1989. Sensitive determination of circulating anodic antigen in Schistosoma mansoni infected individuals by an enzyme-linked immunosorbent assay using monoclonal antibodies. Am J Trop Med Hyg 40: 268-272.

Dillon GP, Feltwell T, Skelton JP, Ashton PD, Coulson PS, Quail MA, Nikolaidou-Katsaridou N, Wilson RA, Ivens AC 2006. Microarray analysis identifies genes preferentially expressed in the lung schistosomulum of Schistosoma mansoni. Int J Parasitol 36: 1-8.

Eberl M, Langermans JA, Vervenne RA, Nyame AK, Cummings RD, Thomas AW, Coulson PS, Wilson RA 2001. Antibodies to glycans dominate the host response to schistosome larvae and eggs: is their role protective or subversive? $J$ Infect Dis 183: 1238-1247.

Farah IO, Mola PW, Kariuki TM, Nyindo M, Blanton RE, King CL 2000. Repeated exposure induces periportal fibrosis in Schistosoma mansoni-infected baboons: role of TGFbeta and IL-4. J Immunol 164: 5337-5343.

Farah IO, Nyindo M, Suleman MA, Nyaundi J, Kariuki TM, Blanton RE, Elson LH, King CL 1997. Schistosoma mansoni: development and modulation of the granuloma after or multiple exposures in the baboon (Papio cynocephalus anubis). Exp Parasitol 86: 93-101.

Kariuki TM, Farah IO, Yole DS, Mwenda JM, van Dam GJ, Deelder AM, Wilson RA, Coulson PS 2004. Parameters of the attenuated schistosome vaccine evaluated in the olive baboon. Infect Immun 72: 5526-5529. 
Kariuki TM, van Dam GJ, Deelder AM, Farah IO, Yole DS, Wilson RA, Coulson PS 2006. Previous or ongoing schistosome infections do not compromise the efficacy of the attenuated cercaria vaccine. Infect Immun In press.

King CH, Dickman K, Tisch DJ 2005. Reassessment of the cost of chronic helmintic infection: a meta-analysis of disability-related outcomes in endemic schistosomiasis. Lancet 365: 1561-1569.

King CL 2001. Initiation and regulation of disease in schistosomiasis. In AAF Mahmoud, Schistosomiasis, Vol. 3, Imperial College Press, London, p. 213-264.

McHugh SM, Coulson PS, Wilson RA 1987. Pathologically induced alterations in the dimensions of the hepatic portal vasculature of mice infected with Schistosoma mansoni. Parasitology 94: 69-80.

Nyindo M, Kariuki TM, Mola PW, Farah IO, Elson L, Blanton
RE, King CL 1999. Role of adult worm antigen-specific immunoglobulin E in acquired immunity to Schistosoma mansoni infection in baboons. Infect Immun 67: 636-642.

Polman K, Diakhate MM, Engels D, Nahimana S, van Dam GJ, Falcão Ferreira ST, Deelder AM, Gryseels B 2000. Specificity of circulating antigen detection for schistosomiasis mansoni in Senegal and Burundi. Trop Med Int Health 5: 534-537.

Sturrock RF, Butterworth AE, Houba V, Karamsadkar SD, Kimani R 1978. Schistosoma mansoni in the Kenyan baboon (Papio anubis): the development and predictability of resistance to homologous challenge. Trans $R$ Soc Trop Med Hyg 72: 251-261.

Yole DS, Pemberton R, Reid GD, Wilson RA 1996. Protective immunity to Schistosoma mansoni induced in the olive baboon Papio anubis by the irradiated cercaria vaccine. Parasitology 112: 37-46. 